\title{
Incorporating asymmetric hardening behavior in modeling the cold expansion process of AZ31B sheet
}

\author{
Sasan Faghih, Seyed Behzad Behravesh, Hamid Jahed \\ Mechanical and Mechatronics Engineering Department, University of Waterloo \\ Waterloo, Ontario
}

\begin{abstract}
The Variable Material Properties (VMP) method of elastoplastic analysis for axisymmetric problems is extended to the applications of the cold expansion problem for magnesium sheets exhibiting asymmetric hardening behavior. To investigate the effect of hardening asymmetry in the stressstrain results, a detailed 3D finite element (FE) model with symmetric material properties was also generated for the cold expansion problem. Comparison between the results of the VMP and the FE model revealed the significant effect of hardening asymmetry.
\end{abstract}

Keywords- Asymmetric hardening; VMP; Cold expansion; Finite element

\section{INTRODUCTION}

Lightweighting, as an effective approach to improve fuel efficiency and reduce greenhouse gasses emissions, has been a major strategy in the transportation industry [1]. Magnesium, as the lightest structural metal, has been a candidate for replacing the existing heavier materials in this industry. Magnesium application in the aerospace industry goes back to early aircraft development in 1930s, mostly for non-load bearing components, such as gearbox casing [2]. Recently, it has been considered also for structural components [3].

In aerospace structures, fastened joints are the most common assembly technique [4]. Rivet joints, causing stress concentration in structures, are highly susceptible to fatigue failure [5]. One of the approaches to improve the fatigue life is to create compressive residual stress around the hole by plastic expansion. There are several ways of expanding the hole, such as hole edge expansion, ball expansion and tapered mandrel. Due to the surface damage caused by these methods [4], they have been replaced by a more advanced method called split sleeve cold expansion. This method, which consists of pulling a tapered mandrel covered with split sleeve through the hole [6], is now widely used in aerospace industry as the fatigue life improvement technique [7]. From the design point of view, determination of the residual stress due to cold expansion is key to estimation of fatigue life. Such stresses have been shown to strictly depend on the stress-strain material model employed in the calculation/simulation [8]. Current calculation/simulation models for cold expansion process, either finite element models [9], [10] or analytical models [11], [12] are based on simple material models, which can only consider symmetric yield and hardening behaviors. The variable material property (VMP), with capability of considering actual material behavior [13][15], is proven to be able to consider the asymmetric behavior ([16]).

Wrought magnesium alloys, show asymmetric behavior, which is defined as different yield strength and hardening behavior under tension and compression. Such asymmetry is arising from twinning deformation [17] and is different from hydrostatic stresses-associated strength-differential effect seen in high strength steels [18]. In addition, wrought magnesium alloys exhibit anisotropy, i.e., the material behavior varies in different orientations. It has been shown that in rolled AZ31B, asymmetric behavior is dominant, and anisotropy is not significant [19].

In this paper, a simple numerical method based on the VMP approach [20], with the capability of considering the asymmetric hardening behavior, is implemented to model the cold expansion process. A detailed 3D finite element model assuming symmetric hardening behavior is developed for the same problem. A comparison is made between the asymmetric solution from the VMP method and the symmetric solution from the FE analysis, to investigate the effect of material symmetry.

\section{MATERIAL AND MODELING}

\section{A. Split sleeve cold expansion process}

In split sleeve cold expansion process, a tapered mandrel surrounded with a split sleeve, is pulled through the hole. Since the summation of the maximum diameter of the mandrel and split sleeve thickness is greater than the initial hole diameter, the hole expands. Upon removal of the mandrel elastic region around the hole springs back, which creates compressive residual stress around the hole. Schematic illustration of the split sleeve cold expansion process is shown in Fig. 1. Details of the geometry of different parts that are used in this study is given in Table 1. Sheet material is AZ31B with properties given in [21]. 




Figure 1. Schematic illustration of the split sleeve cold expansion process

Table 1. Geometry of different parts used in the cold expansion model

\begin{tabular}{|c|c|c|}
\hline Part & dimension & size $(\mathbf{m m})$ \\
\hline Mandrel & Major diameter & 5.84 \\
\hline Split sleeve & Thickness & 0.20 \\
\hline Workpiece & Thickness & 4.00 \\
\hline Workpiece & Initial hole diameter & 6.00 \\
\hline
\end{tabular}

B. $V M P$ approach

First, a brief review of the VMP method is presented here. Total strain in a body subjected to general force can be written as:

$$
\varepsilon_{i j}=\varepsilon_{i j}^{e}+\varepsilon_{i j}^{p}
$$

in which $\varepsilon, \varepsilon^{\mathrm{e}}$, and $\varepsilon^{\mathrm{p}}$ stand for total strain, elastic strain, and plastic strain, respectively. According to Hook's law:

$$
\varepsilon_{i j}{ }^{e}=\frac{1+v}{v} \sigma_{i j}-\frac{v}{E} \sigma_{k k} \delta_{i j}
$$

where $v, E, \delta, \sigma$ are Poisson's ratio, Young's modulus, Kronecker delta, and stress components. According to the Hencky's total deformation theory:

$$
\begin{gathered}
\varepsilon_{i j}^{p}=\phi S_{i j} \\
S_{i j}=\sigma_{i j}-\frac{1}{3} \sigma_{k k} \delta_{i j} \\
\phi=\frac{3}{2} \frac{\varepsilon_{e q}^{p}}{\sigma_{e q}}
\end{gathered}
$$

where $\mathrm{S}$ stands for deviatoric stress and $\phi$ is a scalar value, obtained from uniaxial stress-strain curve. Combining "(3)"and “(2)” with “(1)" results in:

$$
\begin{gathered}
\varepsilon_{i j}=\left(\frac{1+v}{E}+\phi\right) \sigma_{i j}-\left(\frac{v}{E}+\frac{\phi}{3}\right) \sigma_{k k} \delta_{i j} \\
\varepsilon_{i j}=\frac{1+v_{e f f}}{E_{e f f}} \sigma_{i j}-\frac{v_{e f f}}{E_{e f f}} \sigma_{k k} \delta_{i j} \\
E_{e f f}=\frac{3 E}{3+2 E \phi} \\
v_{e f f}=\frac{3 v+E \phi}{3+2 E \phi}
\end{gathered}
$$

In which, the "eff" subscript stands for the effective value.

To solve an axisymmetric boundary value problem, such as a cylinder subjected to an internal pressure, Fig. 2, the body is discretized into a finite number of annuli. The VMP method, being an iterative solution, starts with a fully elastic solution in the first iteration. Then, the equivalent stress is calculated for all the elements. For the elements where the equivalent stress is higher than the yield strength of the material, the values of $v_{\text {eff }}, E_{\text {eff }}$ are updated according to Equation (4), which are used in the next solution iteration. The effective Young's modulus at each element is found from the projection method, illustrated in Fig. 2(b). According to the projection method, the effective Young's modulus is the secant modulus at the strain value obtained in the previous iteration, point $b^{\prime}$ in Fig. 2(b). Eliminating $\phi$ in Equation (4) gives the effective Poisson's ratio. Then, the elastic solution in each iteration is obtained from the well-known Lame's solution in the plane stress condition [20]. This procedure is repeated until the equivalent stress and strain values for all the elements match the uniaxial stress-strain curve. In case of the cold expansion problem, the process is modeled as a cylinder, with inside diameter equal to the hole diameter and outside diameter which is five times the hole diameter, subjected to radial displacement at inner radius.

As mentioned earlier, in magnesium alloys uniaxial tension and compression curves are not the same. To consider the asymmetric behavior, the Cazacu-Barlat yield criterion [22] was implemented to calculate the equivalent stress in the VMP method iterations. This criterion is given by:



Figure 2. (a) Pressurized thick-walled cylinder discretized into finite number of annuli, (b) demonstraion of the projection method

$$
\begin{gathered}
\sigma_{e q}=A\left(J_{2}^{\frac{3}{2}}-c J_{3}\right)^{\frac{1}{3}} \\
c=\frac{3 \sqrt{3}}{2}\left(\frac{\sigma_{y t}^{3}-\sigma_{y c}^{3}}{\sigma_{y t}^{3}+\sigma_{y c}^{3}}\right) \\
A=3\left(3^{\frac{3}{2}}-2 c\right)^{\frac{-1}{3}}
\end{gathered}
$$


In which, $\sigma_{\mathrm{yt}}, \sigma_{\mathrm{yc}}, \mathbf{J}_{2}$ and $\mathbf{J}_{3}$ are tension and compression yield strenghts, second and third invariants of deviatoric stress tensor, respectively. For the case of symmetric materials, i.e. $\sigma_{\mathrm{yt}}=\sigma_{\mathrm{yc}}$, this yield criterion reduces to the Von-Mises criterion. The asymmetry parameter, $\mathrm{c}$, determines the shape of the yield locus. The parameter $\mathrm{c}$ at a given plastic strain is obtained from the ratio of stresses on the uniaxial tension and compression curves (Fig. 3) in that plastic strain. Therefore, the shape of the yield locus for the Cazacu-Barlat criterion evolves during the plastic deformation, while it is fixed for the Von-Mises criterion. It is noteworthy that for both the symmetric (Von-Mises criterion) and asymmetric (Cazacu-Barlat criterion) solutions, only the uniaxial tension curve is used in the projection method. For the asymmetric solution, the evolution of the parameter $\mathrm{c}$ accounts for the compression hardening curve being different than the tension curve.

To obtain the residual stress field created by cold expansion two steps of loading and unloading are required. The unloading solution is similar to the loading part, but now each element has a specific unloading behavior defined by the level of loading strain. For the unloading phase, a pressure with the same value as the pressure at the end of the loading step with an opposite sign is applied to the inner radius of the cylinder. The negative pressure in the unloading step simulates the removal of the mandrel. Since in the Cazacu-Barlat yield criteria, tension and compression curves need to be determined. For the unloading solution, second part of the tension-compression curve (Fig. 4b) is considered as tensile curve. Likewise, the second part of the compression-tension (Fig. 4a) is considered as compression curve as the input for the VMP method. Simple interpolation method was used to generate the curves that are in between unloading curves shown in Fig. 4. After solving the unloading part, the residual stress field was obtained by:

$$
\begin{gathered}
\sigma_{\text {Radial }}^{\text {Res }}=\sigma_{\text {Radial }}^{\text {load }}+\sigma_{\text {Radial }}^{\text {Unload }} \\
\sigma_{\text {Hoop }}^{\text {Res }}=\sigma_{\text {Hoop }}^{\text {load }}+\sigma_{\text {Hoop }}^{\text {Unload }}
\end{gathered}
$$

In which, $\sigma^{\text {Res }}, \sigma^{\text {Load }}, \sigma^{\text {Unload }}$ are residual stress, stress in the loading step, and the stress in the unloading step, respectively.



Figure 3. Uniaxial tension and compression behavior of rolled AZ31B [21]
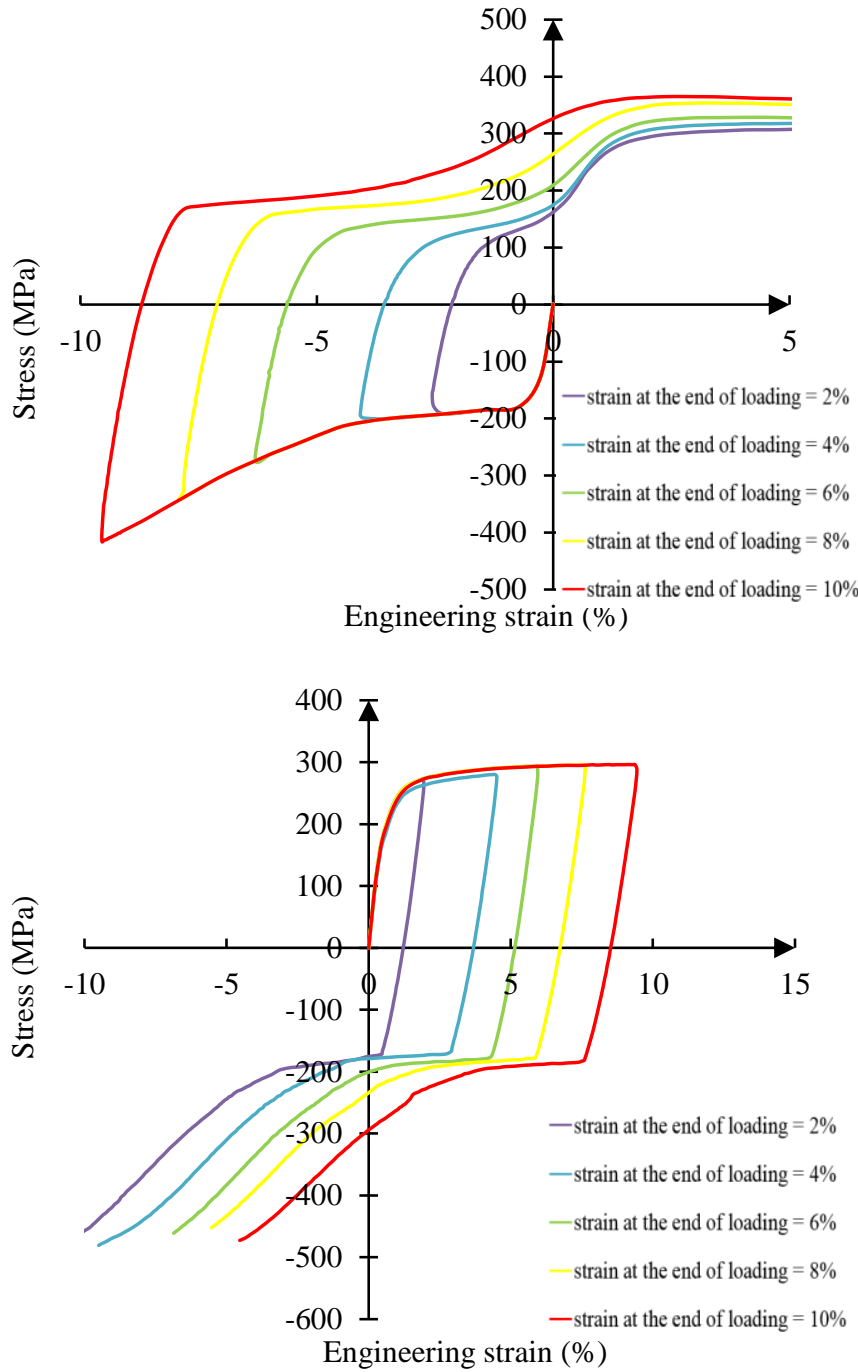

Figure 4. (a) Compression-tension and (b) tension-compression behavior for rolled AZ31B ([21],[23])

\section{Finite element}

ABAQUS finite element software was used in this study to simulate the cold expansion process (Fig. 5). In order to apply a more realistic boundary condition, the support of the workpiece was also included in the model. Owing to the symmetry, only half of the structure was modeled. Boundary conditions of the model are shown in Fig. 6. On the X-Y plane, movement of the external edges of the workpiece and the support were constrained. Mandrel was considered rigid, while the split sleeve and steel support were linear elastic material with the Young's modulus of $200 \mathrm{GPa}$ and Poison's ratio of 0.3 . The workpiece followed the tensile curve (Fig. 3) for the loading step, and the hardening behavior was assumed to be isotropic for the unloading step. Three surface-to-surface contacts were applied to the model: between mandrel and split sleeve, split sleeve and workpiece, and workpiece and support. They all were considered as frictionless contact. Hexahedral linear brick elements with 8 nodes and one integration point, C3D8R, were used to construct the workpiece. Because the most important region of the workpiece is the area close to the hole, the mesh size was biased towards the hole. 




Figure 5. Assembly view of the 3D model

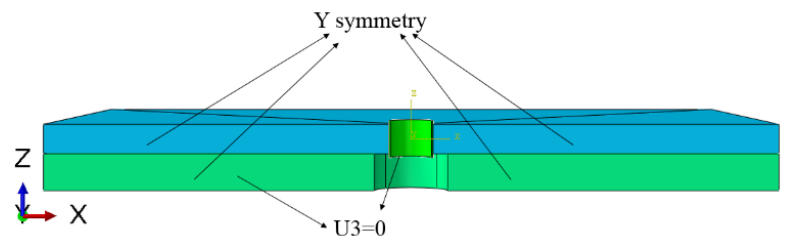

Figure 6. Boundary condition of the model

III. RESULT AND DISCUSSION

To validate the results of the VMP solution, a comparison between the VMP and the ABAQUS results using only the tension curve and assuming isotropic hardening in both VMP and ABAQUS was made. Fig. 7 and Fig. 8 show the loading and residual stress distributions, respectively. The results from ABAQUS is for the mid-plane of the workpiece and far from the position of split. As expected, the results are in good agreement. The difference can be attributed to the fact that ABAQUS is a 3D model that considers more detail such as effect of support for workpiece, contact between mandrel and split sleeve and workpiece, while the VMP models the process as a 2D uniform expansion. Fig. 9 and Fig. 10 show the effect of considering asymmetric behavior in loading and unloading in stress distribution.



Figure 7. Hoop and radial stress distribution at the end of loading assuming symmetric hardening behavior

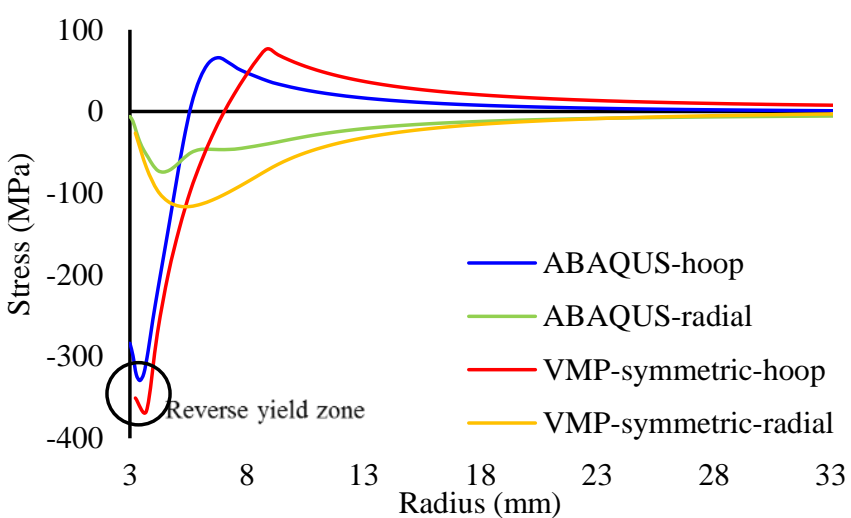

Figure 8. Residual hoop and radial stress distribution assuming symmetric hardening behavior

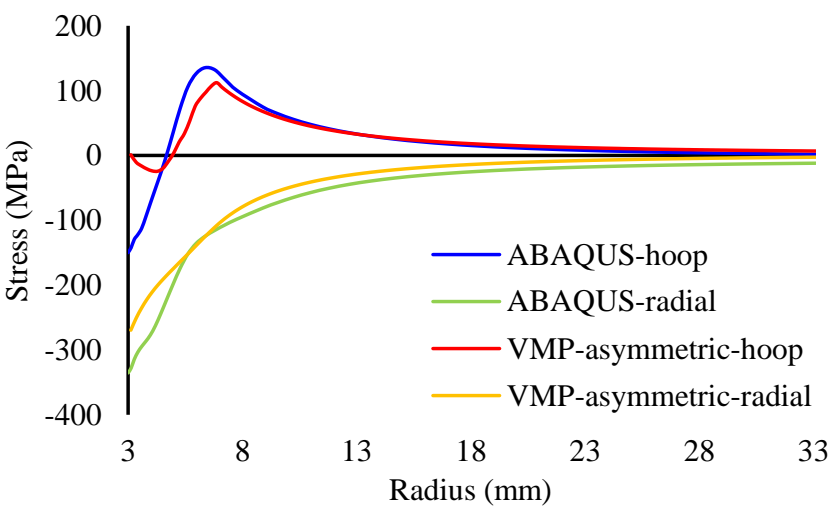

Figure 9. Hoop and radial stress distribution at the end of loading assuming symmetric hardening in the ABAQUS and asymmetric hardening in the VMP

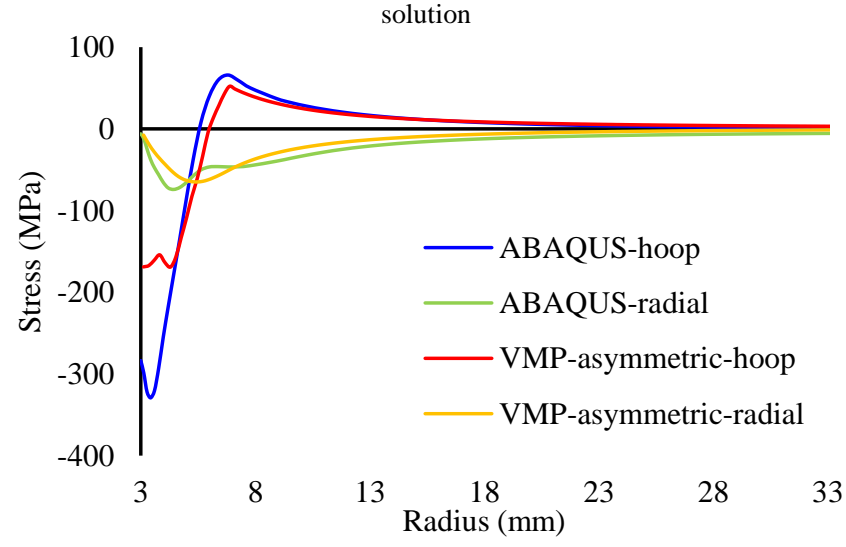

Figure 10. Residual hoop and radial stress distribution assuming symmetric hardening in the ABAQUS and asymmetric hardening in the VMP solution

In Fig. 9 and Fig. 10, the results of VMP is obtained with asymmetric material model, while in ABAQUS, symmetric behavior was assumed.

Hoop stress distributions close to the hole in loading and unloading steps were significantly different between the symmetric ABAQUS and asymmetric VMP solutions. Close to hole, which is the area of interest for the cold expansion problem, the VMP predicts the value of hoop stress to be close to zero, Fig. 9. Likewise, the residual hoop stress distribution is meaningfully different. ABAQUS predicts the reverse yield zone to be close to the hole, while Asymmetric VMP model predicts the reverse yield zone to be larger, i.e. farther from the hole (Fig. 10). This can be explained by different unloading 
curves used in ABAQUS and VMP. VMP considers actual material unloading behavior (Fig. 4), in which reverse yield in the unloading part, happens at lower stress, while in ABAQUS model, the unloading curve is constructed using the isotropic hardening assumption. Moreover, considering the actual unloading behavior of the material by VMP and assuming isotropic hardening rule by ABAQUS, also contributed to the difference between the calculated residual stress field. The difference in residual stress distribution is also due to difference in the hoop stress distribution in the loading step. The plastic strain obtained from VMP around the hole during the loading step is shown in Fig. 11, for symmetric and asymmetric behavior. The larger plastic strain in the asymmetric solution is due to compression behavior accounted for in this solution. Because the yield stress in compression is smaller than that in tension (Fig. 3), it is expected that the plastic strain close to hole to be higher in the asymmetric model compared to the symmetric model.

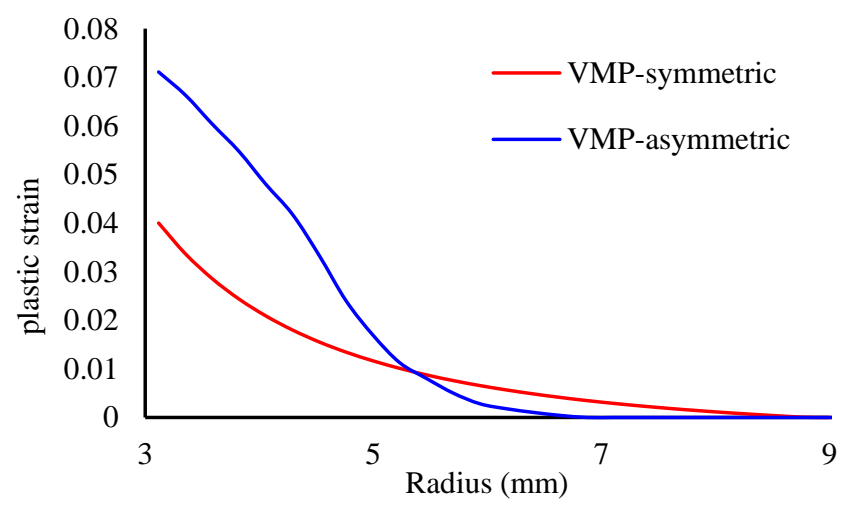

Figure 11. Plastic strain around the hole at the end of loading

\section{CONCLUSTION}

The cold expansion process in AZ31B wrought magnesium alloy has been modeled using the finite element and the VMP methods. The asymmetric hardening behavior of AZ31B was captured by the VMP, but the finite element method did not account for the asymmetric hardening behavior. Following conclusions can be drawn from this study:

- The VMP method is capable of modeling the cold expansion process. For symmetric materials, the results of this simple 2D model are in agreement with predictions of the 3D finite element model in the mid-plane.

- Asymmetric hardening behavior of materials can significantly affect the stress distribution in loading and unloading steps of the cold expansion process.

- Hoop stress distribution is significantly different in the asymmetric model, while the radial stress is almost not affected.

\section{REFERENCES}

[1] J. P. Immarigeon, R. T. Holt, A. K. Koul, L. Zhao, W. Wallace, and J. C. Beddoes, "Lightweight materials for aircraft applications," Mater. Charact., vol. 35, no. 1, pp. 41-67, 1995.

L. Ostrovsky and Y. Henn, "Present state and future of magnesium application in aerospace industry," Int. Conf. "New Challenges Aeronaut. ASTEC 07, pp. 1-5, 2007.

[3] G. Song and a. Atrens, "Understanding Magnesium Corrosion-A Framework for Improved Alloy Performance," Adv. Eng. Mater., vol. 5, no. 12, pp. 837-858, 2003.

[4] Y. Fu, E. Ge, H. Su, J. Xu, and R. Li, "Cold expansion technology of connection holes in aircraft structures: A review and prospect," Chinese J. Aeronaut., vol. 28, no. 4, pp. 961-973, 2015.

[5] G. L. Kulak, J. W. Fisher, and J. H. A. Struik, Guide to design criteria for bolted and riveted joints, vol. 15, no. 1. 1988.

[6] D. A. Clark and W. S. Johnson, "Temperature effects on fatigue performance of cold expanded holes in 7050-T7451 aluminum alloy," Int. J. Fatigue, vol. 25, no. 2, pp. 159-165, 2003.

V. Achard, A. Daidie, M. Paredes, and C. Chirol, "Optimization of the Cold Expansion Process for Titanium Holes," Adv. Eng. Mater., vol. 19, no. 6, pp. 1-13, 2017.

A. A. Garcia-Granada, V. Lacarac, D. J. Smith, M. J. Pavier, R. Cook, and P. Holdway, "3D residual stresses around cold expanded holes in a new creep resistant aluminium alloy," Trans. Eng. Sci., vol. 25, pp. 103-116, 1999.

V. Nigrelli and S. Pasta, "Finite-element simulation of residual stress induced by split-sleeve cold-expansion process of holes," J. Mater. Process. Technol., vol. 205, no. 1-3, pp. 290-296, 2008.

S. J. Houghton and S. K. Campbell, "Identifying the residual stress field developed by hole cold expansion using finite element analysis," Fatigue Fract. Eng. Mater. Struct., vol. 35, no. 1, pp. 74-83, 2012.

Rich and Impellizzeri, "Fatigue analysis of cold-worked and interference fit fastener holes," ASTM Spec Tech Publ 637, pp. 153 $175,1977$.

G. S. Wang, "An elastic-plastic solution for a normally loaded center hole in a finite circular body," Int. J. Press. Vessel. Pip., vol. 33, no. 4, pp. 269-284, 1988.

[13] H. Jahed, S. B. Lambert, and R. N. Dubey, "Variable material property method in the analysis of cold-worked fastener holes," $J$. Strain Anal. Eng. Des., vol. 35, no. 2, pp. 137-142, 2000.

[14] H. Jahed, B. A. Moghadam, and M. Shambooli, "Re-Autofrettage," J. Press. Vessel Technol., vol. 128, no. 2, p. 223, 2006.

[15] S. Faghih, H. Jahed, and S. B. Behravesh, "Variable Material Properties (VMP) Approach: A Review on Twenty Years of Progress," J. Press. Vessel Technol., 2018.

[16] M. A. Khayamian, B. Behravesh, and H. Jahed, "Incorporation ofasymmetric yield and hardening behaviour in axisymmetric elastoplastic problems," Mater. Des., vol. 99, pp. 490-499, 2016.

J. Albinmousa, H. Jahed, and S. Lambert, "Cyclic axial and cyclic torsional behaviour of extruded AZ31B magnesium alloy," Int. J. Fatigue, vol. 33, no. 11, pp. 1403-1416, 2011.

[18] J. Casey and H. Jahedmotlagh, "The strength-differential effect in plasticity,” Int. J. Solids Struct., vol. 20, no. 4, pp. 377-393, 1984.

[19] S. B. Behravesh, "Fatigue Characterization and Cyclic Plasticity Modeling of Magnesium Spot-Welds," University of Waterloo, 2013. H. Jahed and R. N. Dubey, "An axisymmetric method of elasticplastic analysis capable of predicting residual stress field," J. Press. 
Vessel Technol., vol. 119, no. 3, pp. 264-273, 1997.

[21] S. B. Behravesh, H. Jahed, and S. Lambert, "Characterization of magnesium spot welds under tensile and cyclic loadings," Mater. Des., vol. 32, no. 10, pp. 4890-4900, 2011.

[22] O. Cazacu and F. Barlat, "A criterion for description of anisotropy and yield differential effects in pressure-insensitive metals," Int. J. Plast., vol. 20, no. 11 SPEC. ISS., pp. 2027-2045, 2004.

[23] A. Yazdanmehr and H. Jahed, "Large strain compression-tension behavior of AZ31B rolled sheet in the rolling direction," UNPUBLISHED. 